\title{
Primary Care Physician Perspectives on Caring for Adult Survivors of Hematologic Malignancies and Hematopoietic Cell Transplantation
}

\author{
Shylaja Mani, MD¹, Nandita Khera, MD, MPH${ }^{2}$, Lisa Rybicki, MS ${ }^{3}$, Naimisha Marneni, MD ${ }^{4}$, \\ Hetty Carraway, MD ${ }^{4}$, Halle Moore, MD4, Helen Whited, MD², Navneet S Majhail, MD, MS ${ }^{4}$ \\ ${ }^{1}$ Department of Hematology and Oncology, Ohio State University, Columbus, $\mathrm{OH}$; \\ ${ }^{2}$ Department of Hematology and Oncology, Mayo Clinic, Phoenix, AZ; \\ ${ }^{3}$ Department of Quantitative Health Sciences, Cleveland Clinic, Cleveland, $\mathrm{OH}$; \\ ${ }^{4}$ Department of Hematology and Oncology, Cleveland Clinic, Cleveland, $\mathrm{OH}$
}

\section{Abstract}

Background: Primary care physicians (PCPs) may face barriers to caring for hematologic malignancy and hematopoietic cell transplantation (HCT) survivors.

\begin{abstract}
Methods: A web-based survey consisting of 40 questions and 2 case scenarios was administered to 302 PCPs at two large integrated healthcare systems. The questionnaire assessed perceived barriers to delivery of care to hematologic malignancy/HCT survivors, resources available to care for cancer survivors, practices for care coordination with hematologist-oncologists, and preferred models of care delivery.
\end{abstract}

Results: Overall response rate was $30 \%(\mathrm{~N}=86)$. PCPs reported several barriers such as lack of resources to facilitate care (69\%), lack of awareness of screening/prevention guidelines (55\%) and psychosocial needs of survivors (65\%), inadequate time (65\%), and patient preference to follow up with their oncologists $(66 \%)$. They expressed confidence in caring for general medical issues $(84 \%)$ and general cancer screening $(73 \%)$, but preferred oncologists manage cancer related medical issues (42\%), screen for cancer recurrence (52\%) and secondary cancers (55\%). In multivariable analysis, PCPs who had previously cared for larger number of hematologic malignancy/HCT survivors and those further out since graduation from medical school had greater confidence in managing cancer related medical issues.

Conclusions: PCPs report several barriers in providing care to hematologic malignancy/HCT survivors. Clinical experience with this patient population is associated with greater confidence in providing survivorship care. Several barriers identified by PCPs in providing survivorship care to

Corresponding Author: Navneet S Majhail, MD, MS, Blood and Marrow Transplant Program, Cleveland Clinic, 9500 Euclid Ave, CA60, Cleveland, OH 44195, majhain@ @cf.org, Phone: 216-444-2199.

Author Contributions:

Conception and design: Shylaja Mani, Nandita Khera, Lisa Rybicki, Hetty Carraway, Halle Moore, Helen Whited, Navneet S Majhail. Collection and assembly of data: Shylaja Mani, Nandita Khera, Lisa Rybicki, Helen Whited, Navneet S Majhail.

Data analysis and interpretation: All authors

Manuscript writing and final approval of manuscript: All authors

Conflict of Interest: None of the authors has any financial conflicts of interest to disclose in relationship to this study. 
hematologic malignancy/HCT survivors are potentially addressable by education, clinical decision support tools and guidelines, enhancing clinical experience, and care coordination with hematologist-oncologists.

\section{MICRO-ABSTRACT}

In a survey of primary care physicians at two large integrated healthcare systems, several barriers to providing care to hematologic malignancy/hematopoietic cell transplant survivors were identified, several of which are potentially addressable by education, enhancing clinical experience, and care coordination with hematologist-oncologists. Clinical experience with this patient population is associated with greater confidence in providing survivorship care.

\section{Keywords}

Cancer survivor; Hematologic malignancy; Hematopoietic cell transplantation; Late complications; Care delivery; Models of care; Primary care physicians

\section{INTRODUCTION}

Advances in treatment for hematologic malignancies and hematopoietic cell transplantation (HCT) has led to improved survival and increasing number of adult long-term survivors of diseases such as acute and chronic leukemia, myelodysplastic syndromes, lymphoma and multiple myeloma ${ }^{1-7}$. These survivors are at risk for long-term complications including late infections, secondary cancers, psychosocial stress, graft-versus-host disease and are hence considered moderate to high risk groups in the risk stratified approach to survivorship ${ }^{8}$. With the exception of Hodgkin lymphoma, long-term mortality and late effects have not been systematically studied in survivors of hematologic malignancies ${ }^{9}$. However, extensive research on HCT survivors shows that their relative mortality rates continue to remain higher than expected compared to their general population peers through at least 15 years after transplantation ${ }^{10-12}$. Excess mortality is primarily driven by late complications and guidelines recommend lifelong follow up with regular evaluations for their screening and prevention $^{9,13,14}$.

Cancer patients who are in remission long-term after their treatment frequently receive healthcare through their primary care physicians (PCPs) and are less likely to be under the care of cancer centers and hematologist-oncologists ${ }^{15,16,17}$. However, several PCP barriers to providing survivorship care have been identified, such as lack of training and uncertainty about general preventive care responsibilities ${ }^{18-20}$. These barriers may be accentuated and different for hematologic malignancy and HCT survivors, given the rarity of their diagnoses, the highly-specialized nature of treatments that these patients receive, and their associated complications. With this background, we conducted a survey of PCPs at two institutions to identify the knowledge gaps and perceived barriers in the management of survivorship issues in this patient population, and to identify their current practices, utilization of available resources and coordination of care with specialists. 


\section{METHODS}

\subsection{Study Sites}

A cross-sectional survey was administered to Internal Medicine and Family Practice physicians caring for adult patients and practicing through the Cleveland Clinic in Cleveland, Ohio and the Mayo Clinic in Scottsdale, Arizona. Both institutions have a similar model with a main tertiary care academic medical center and satellite clinics in the regional area, with PCPs employed in an integrated health system. Care for hematologic malignancies and HCT is mainly provided through hematologist-oncologists at cancer centers located at their main tertiary care hospitals. Most long-term survivorship care for hematologic malignancies and HCT survivors is provided through hematologist-oncologists with varying degree of involvement by patient's PCPs. Patients are typically treated at the main campus location, especially allogeneic HCT recipients who are also required to stay locally through at least day +100 after transplantation. The frequency of subsequent posttransplant follow up varies based on patient needs, underlying diagnosis and presence of post-transplant complications, although allogeneic HCT recipients are followed for life. Routine interactions between the two groups of physicians varies, depends on the clinical status of the patient, and is usually focused around sharing of patient notes and information through electronic medical record at each site.

\subsection{Survey Development}

We adapted a previously published survey instrument that assessed physician knowledge, beliefs and practices regarding the care of breast and colon cancer survivors for our study ${ }^{18}$. Questions specific for hematologic malignancy and HCT survivors were added to the questionnaire. Broadly, the survey addressed PCP knowledge of long-term complications and recommended preventive follow-up care for survivors of hematological malignancies and HCT, their perceived barriers and limitations to delivery of care to this patient population, resources needed and currently available to provide care to cancer survivors in general, their current practice for care coordination with hematology-oncology providers for cancer survivors in general, and their preferred models of care delivery for hematologic malignancy and HCT survivors. Additionally, case vignettes were included to understand the willingness of PCPs to provide routine medical care for patients with varying complexity of survivorship care needs.

The survey instrument was piloted with 5 PCPs at the Cleveland Clinic to address any redundancy in its content, to obtain feedback on case vignettes, and to provide an estimate of the time for completion. The final instrument consisted of 40 questions. Responses regarding perceptions and barriers were organized in an ordinal scale and were "Never", "Rarely", "Often" and "Always". Questions about resource utilization were categorized as "Not helpful", "Somewhat helpful" and "Very helpful". Based on pilot study feedback, two case scenarios were chosen for inclusion in the final survey and represented patient situations that were most likely to be seen independently by PCPs and required minimal active ongoing care by hematologist-oncologists. First scenario represented a 40-year old female, three years after completion of therapy for acute myeloid leukemia (AML) with an uncomplicated treatment course and asked respondents to describe their confidence in independently 
providing care for general medical issues, cancer-related medical issues, screening for cancer recurrence and secondary cancers. Respondents could answer on a Likert scale as "Not at all confident", "Somewhat confident" and "Very confident". The second scenario represented an identical patient, with the exception that the patient had also received an allogeneic HLA-identical sibling donor transplant but had no post-transplant complications.

Practice information gathered from respondents included number of hematologic malignancy and HCT survivors seen in their practice within the past 5 years, primary specialty, site of practice, present involvement in teaching medical students or residents, number of patients seen in a typical week, and year of medical school graduation.

\subsection{Survey Administration}

The final survey was administered as a web-based survey using REDCap at both institutions and took an estimated 10-15 minutes to complete. An initial email invite with a link to the survey was sent to PCPs on their institutional email accounts and was followed by two reminders 2 weeks apart. Respondents were instructed to consider patients with hematologic malignancies and HCT recipients (acute and chronic leukemia, myelodysplastic syndrome, lymphoma, and myeloma) who had completed active treatments, were in remission, and did not require intensive monitoring by hematologist-oncologist for at least one year or more following completion of therapy. Twenty respondents were randomly chosen to receive a Visa gift card of $\$ 50$ at the Cleveland Clinic and 15 respondents were randomly chosen to receive a Visa gift card of $\$ 25$ at the Mayo Clinic. The study was conducted under guidance of Institutional Review Boards of both participating institutions.

\subsection{Statistical Analysis}

Data from REDCap were exported into SAS for analysis. For analysis, responses of "Never" and "Rarely" were combined as one category and "Often" and "Always" were combined as a second category. For questions inquiring about resources, no one answered "Not helpful" and the analysis considered "Somewhat helpful" versus "Very helpful". Confidence questions for case vignettes were analyzed as "Not confident" versus "Somewhat confident" versus "Very confident". For ordinal categorical PCP characteristics (number of survivors cared for, number of patients seen, years since graduation), associations with binary responses were analyzed with Cochran Armitage trend test and associations with ordinal responses were analyzed with Cochran-Mantel-Haenzel correlation test; both assess trends across levels of PCP characteristics. For binary categorical PCP characteristics (primary specialty, practice site, teaching), associations with binary responses were analyzed with Chi-square test and associations with ordinal responses were analyzed with CochranMantel-Haenzel mean score test. We also performed an analysis to evaluate the association of PCP characteristics with their confidence in managing various scenarios in the two case vignettes. First, ordinal logistic regression was done for the four questions associated with each of the two vignettes. Then stepwise logistic regression analysis with a variable entry criterion of $p<0.10$ and a variable retention criterion of $p<0.05$ was used to identify multivariable prognostic factors. Results are summarized as odds ratio (OR), 95\% confidence interval (CI), and p-value. 


\section{RESULTS}

\subsection{Physician Characteristics}

A total of 302 PCPs were invited to participate in the survey, including 256 employed at the Cleveland Clinic and 37 at Mayo Clinic, Arizona. There were 59 respondents from Cleveland Clinic and 27 from Mayo Clinic with an overall response rate of 29\%. The practice patterns of the 86 respondents are outlined in table 1 .

\subsection{Responses to domains assessed by survey}

Among respondents, 54\% indicated that they "often/always" received comprehensive treatment summary and 75\% "often/always" received periodic updates from the hematologist-oncologist. Overall, 63\% of the PCPs had no difficulty transferring patient care responsibilities with the hematologist-oncologist and only $45 \%$ discussed screening for cancer recurrence, secondary cancers or late effects with the patient.

PCPs reported several barriers in providing care for cancer survivors. The most common physician related barrier was the lack of resources to facilitate survivor care $(69 \%)$ and the most common patient related barrier was the lack of clarity by patients on who to approach for care if health issues arise (77\%). Other barriers noted are elucidated in fig. 1a and fig. 1b.

PCPs preference for providing care for hematologic malignancy and HCT survivors regarding general medical and cancer-related medical issues, psychosocial and fertility issues, screening for cancer recurrence, general and secondary cancers either independently or by sharing responsibility with the hematologist-oncologist are depicted in fig. 1c. Resources reported to be "very helpful" for providing survivor care included survivor care plan (86\%), treatment summary (86\%), structured follow-up plan (87\%) and EMR implemented guidelines (78\%). Fig. 2a and 2b summarize self-rated confidence responses of PCPs to the questions in the AML chemotherapy only and HCT case vignettes.

\subsection{Associations between responses and PCP characteristics}

PCPs who had cared for a greater number of hematologic malignancy/HCT survivors in the past five years were more likely to report that they "often/always" received a comprehensive treatment summary, received periodic updates, and discussed screening/late effects with the patient. Furthermore, they were more likely to prefer to be involved with screening for cancer recurrence, general and secondary cancers, and management of fertility issues. Likewise, there was higher level of confidence in managing cancer-related medical issues in chemotherapy only case scenario and in managing general medical issues or cancer-related medical issues in HCT case scenario. There was also increased confidence in screening for cancer recurrence and secondary cancers in both cases (Table 2).

As number of survivors cared for in the past five years increased, there were decreases in the proportion of PCP's who "often/always" had issues with inadequate resources to facilitate survivor care or inadequate communication with the hematologist-oncologist. On a related note, as number of patients seen in a week increased, there were decreases in the proportion 
of respondents who "often/always" had difficulty transferring patient care responsibilities and who lack awareness of screening/prevention guidelines.

As the years since graduation from medical school increased, respondents reported higher level of confidence in managing cancer-related medical issues and screening for cancer recurrence in both case scenarios. Additionally, as years since graduation increased there were decreases in the proportion of PCPs who "often/always" reported lack of awareness of clinical/psychosocial needs of survivors and of screening/prevention guidelines.

No associations were identified for primary specialty and practice site. However, a higher percentage of responders with teaching responsibilities preferred to handle general medical issues, general cancer screening and management of psychosocial issues. They were also more confident in chemotherapy only case scenario in dealing with general medical issues that may or may not be related to cancer.

In multivariable analysis evaluating the association of PCP characteristics and confidence in managing survivorship issues for the AML chemotherapy only case scenario (Table 3), PCPs who had cared for a greater number of hematologic malignancy/HCT survivors in the past five years were more confident in managing cancer-related medical issues, screening for cancer recurrence and secondary cancers. Likewise, there was increased confidence in handling general medical issues by PCPs with teaching responsibilities and cancer-related medical issues, screening for cancer recurrence by PCPs with increased time since graduation from medical school.

For the HCT case scenario, more years since graduation from medical school was associated with greater confidence in treating cancer-related medical issues and screening for cancer recurrence. Furthermore, PCPs who had cared for a greater number of survivors in the past five years were more confident in managing general medical issues, cancer-related medical issues and screening for secondary cancers.

\section{DISCUSSION}

PCPs are integral to providing comprehensive multidisciplinary survivorship care to cancer patients. Our study has identified several barriers that PCPs face in providing care to hematologic malignancy and HCT survivors. We also identify opportunities for facilitating survivor care through PCPs and areas that may be more appropriate for collaborative care with hematologist-oncologists or other specialists.

In comparison to solid tumors, hematologic cancers are relatively rare and heterogeneous, and patients receive a myriad of treatments and exposures. Only a small proportion of patients with hematologic malignancies go on to receive HCT, which makes this survivor population even smaller. Hence, our survey findings are not surprising given this context where PCPs have a low likelihood of caring for hematologic malignancy and HCT survivors in their practice. The significant findings among others were that about half of PCPs in our study were not aware of the screening guidelines, and psychosocial needs of all cancer survivors and half of them did not discuss screening/late effects with the patient. Overall, this and other observations from our study suggests a need for improved physician education 
and training in survivorship care in our patient population of interest of hematologic malignancy and HCT survivors. Such training could occur through participation in specialized programs, continuing medical education conferences and workshops ${ }^{21}$.

A majority also reported that they lacked the resources including time to provide care. A notable proportion did not face challenges like difficulty transferring care responsibility, insufficient communication with the oncologist and inadequate reimbursement. This is substantially different from earlier studies which report poor care coordination between the oncologist and the $\mathrm{PCP}^{2223}$. A plausible explanation is the practice settings of an integrated cancer center in which the study was conducted, where PCPs and hematologist-oncologists are more likely to be familiar with each other and possibly face less barriers in communicating. PCPs concerns of lack of clarity by the patients on who to approach for care if health issues arise and preference to follow up with their oncologist only suggest a need for patient education as well.

PCPs were willing to assume exclusive responsibility in handling general medical issues that may or may not be related to cancer and general cancer screening for hematologic malignancy and HCT survivors. The finding that the resources that have been "very helpful" for this purpose are survivorship care plan, treatment summary, structured follow-up plan and EMR implemented guidelines echoes The Institute of Medicine's landmark report From Cancer Patient to Cancer Survivor: Lost in Transition findings of the fundamental need for survivorship care plans and improved care coordination to improve the quality of cancer care $^{2425}$. They preferred the oncologists manage cancer-related medical issues, screen for cancer recurrence and secondary cancers. As noted above, this is not surprising given the rarity of this patient population and suggests an area which is amenable to collaborate care between PCPs and hematologist-oncologists.

Not unexpectedly, greater clinical experience, both in general and specifically with hematologic malignancy and HCT survivors, was associated with higher reported confidence in managing general health as well as cancer related issues in this patient population. This finding is in accordance with a study done in internal medicine and family medicine residents which reported greater clinical experience being associated with higher knowledge and confidence in managing cancer related issues in cancer survivors ${ }^{26}$. Although there is no substitute for experiential learning, we do identify PCP groups who may benefit from directed educational interventions on this topic. Future studies can focus on identifying best practices that may be shared with other PCPs to help facilitate survivorship care.

Some limitations of our study need to be acknowledged. The response rate for our survey was relatively low, but not atypical for physician directed surveys. Our PCPs work in an integrated healthcare system that cares for a relatively larger number of hematologic malignancy patients and HCT recipients, and it is possible that barriers faced by PCPs who work in a community practice setting may be different and more pronounced compared to what we observed. We also acknowledge the limitations of a survey that cannot provide a detailed perspective of practice patterns and barriers. There was a difference in response rates between the two sites, which is likely related to the number of PCPs who practice at the two institutions and the resultant engagement to participate in the survey. 
The growing hematologic malignancy and HCT survivor population and the complexities involved in their long-term care heighten the need for more efficient and effective approaches to survivorship care delivery. The current study has highlighted concerns and challenges faced by PCPs in caring for this patient population. Priority should be given to the training of PCPs in survivorship care and earlier involvement during the course of cancer treatment. There is a lack of evidence regarding best practices for survivorship care and developing an evidence-based model of care would be beneficial ${ }^{27}$. The optimal model for providing care to long-term survivors with hematologic malignancies and HCT will require multidisciplinary care with a close partnership between specialty cancer and transplant centers, patient's PCPs and other healthcare providers ${ }^{2}, 5,28$.

\section{ACKNOWLEDGEMENTS}

We are grateful to Donna Corrigan, Research Supervisor, Cleveland Clinic Taussig Cancer Institute for her help with survey administration. This project was supported by the American Society of Hematology HONORS award to Shylaja Mani, MD, during her Internal Medicine residency training at the Cleveland Clinic.

Research Support: Navneet S. Majhail is partially supported by a grant from the National Cancer Institute (R01CA215134). The content is solely the responsibility of the authors and does not necessarily represent the official views of the National Institutes of Health.

\section{REFERENCES}

1. McCarthy PL, Hahn T, Hassebroek A, et al.: Trends in Use of and Survival after Autologous Hematopoietic Cell Transplantation in North America, 1995-2005: Significant Improvement in Survival for Lymphoma and Myeloma during a Period of Increasing Recipient Age. Biol Blood Marrow Transplant 19:1116-1123, 2013 [PubMed: 23660172]

2. Majhail NS, Tao L, Bredeson C, et al.: Prevalence of hematopoietic cell transplant survivors in the United States. Biol Blood Marrow Transplant 19:1498-1501, 2013 [PubMed: 23906634]

3. Majhail NS, Chitphakdithai P, Logan B, et al.: Significant Improvement in Survival after Unrelated Donor Hematopoietic Cell Transplantation in the Recent Era. Biol Blood Marrow Transplant 21:142-150, 2015 [PubMed: 25445638]

4. Hahn T, McCarthy PL, Hassebroek A, et al.: Significant improvement in survival after allogeneic hematopoietic cell transplantation during a period of significantly increased use, older recipient age, and use of unrelated donors. J Clin Oncol 31:2437-2449, 2013 [PubMed: 23715573]

5. Juliusson G, Abrahamsson J, Lazarevic V, et al.: Prevalence and characteristics of survivors from acute myeloid leukemia in Sweden. Leukemia 31:728-731, 2017 [PubMed: 27795559]

6. Kumar SK, Rajkumar SV, Dispenzieri A, et al.: Improved survival in multiple myeloma and the impact of novel therapies. Blood 111:2516-2521, 2015

7. Ward E, Desantis C, Robbins A, et al.: Childhood and Adolescent Cancer Statistics, 2014. Ca Cancer J Clin 64:83-103, 2014 [PubMed: 24488779]

8. Oeffinger KC, McCabe MS: Models for delivering survivorship care. J Clin Oncol 24:5117-5124, 2006 [PubMed: 17093273]

9. Ng AK, van Leeuwen FE: Hodgkin lymphoma: Late effects of treatment and guidelines for surveillance. Semin Hematol 53:209-215, 2016 [PubMed: 27496313]

10. Wingard JR, Majhail NS, Brazauskas R, et al.: Long-term survival and late deaths after allogeneic hematopoietic cell transplantation. J Clin Oncol 29:2230-2239, 2011 [PubMed: 21464398]

11. Majhail NS, Douglas Rizzo J: Surviving the cure: Long term followup of hematopoietic cell transplant recipients. Bone Marrow Transplant 48:1145-1151, 2013 [PubMed: 23292238]

12. Martin PJ, Counts GW, Appelbaum FR, et al.: Life expectancy in patients surviving more than 5 years after hematopoietic cell transplantation. J Clin Oncol 28:1011-1016, 2010 [PubMed: 20065176] 
13. Majhail NS, Rizzo JD, Hahn T, et al.: Pilot study of patient and caregiver out-of-pocket costs of allogeneic hematopoietic cell transplantation. Bone Marrow Transplant 48:865-871, 2013 [PubMed: 23222378]

14. Majhail NS, Rizzo JD, Lee SJ, et al.: Recommended screening and preventive practices for longterm survivors after hematopoietic cell transplantation. Biol Blood Marrow Transpl 18:348-371, 2012

15. Halpern MT, Viswanathan M, Evans TS, et al.: Models of Cancer Survivorship Care: Overview and Summary of Current Evidence. J Oncol Pract 11:e19-e27, 2015 [PubMed: 25205779]

16. Shankar SM, Carter A, Sun C-L, et al.: Health Care Utilization by Adult Long-term Survivors of Hematopoietic Cell T ransplant: Report from the Bone Marrow Transplant Survivor Study. Cancer Epidemiol Biomarkers Prev 16:834-839, 2007 [PubMed: 17416780]

17. Hashmi SK, Bredeson C, Duarte RF, et al.: National Institutes of Health Blood and Marrow Transplant Late Effects Initiative: The Healthcare Delivery Working Group Report. Biol Blood Marrow Transplant 23:717-725, 2017 [PubMed: 27713091]

18. Virgo KS, Lerro CC, Klabunde CN, et al.: Barriers to breast and colorectal cancer survivorship care: Perceptions of primary care physicians and medical oncologists in the United States. J Clin Oncol 31:2322-2336, 2013 [PubMed: 23690429]

19. Smith SL, Wai ES, Alexander C, et al.: Caring for survivors of breast cancer: Perspective of the primary care physician. Curr Oncol 18:218-226, 2011

20. Cheung WY, Aziz N, Noone A-M, et al.: Physician preferences and attitudes regarding different models of cancer survivorship care: a comparison of primary care providers and oncologists. $\mathrm{J}$ Cancer Surviv 7:343-354, 2013 [PubMed: 23526165]

21. Grant M, Economou D, Ferrell B, et al.: Facilitating survivorship program development for health care providers and administrators. J Cancer Surviv 9:180-187, 2015 [PubMed: 25216608]

22. Denzen EM, Preussler JM, Murphy EA, et al.: Tailoring a Survivorship Care Plan: Patient and Provider Preferences for Recipients of Hematopoietic Cell Transplantation. Biol Blood Marrow Transplant, 2018

23. Klabunde CN, Han PKJ, Earle CC, et al.: Physician roles in the cancer-related follow-up care of cancer survivors. Fam Med 45:463-474, 2013 [PubMed: 23846965]

24. Majhail NS, Murphy E, Laud P, et al.: Randomized controlled trial of individualized treatment summary and survivorship care plans for hematopoietic cell transplantation survivors. Haematologica haematol.2018203919, 2018

25. Institute of Medicine, Council NR: From Cancer Patient to Cancer Survivor: Lost in Transition. Washington, DC, The National Academies Press, 2006

26. Susanibar S, Thrush CR, Khatri N, et al.: Cancer survivorship training: a pilot study examining the educational gap in primary care medicine residency programs. J Cancer Surviv 8:565-570, 2014 [PubMed: 24820428]

27. Halpern MT, McCabe MS, Burg MA: The Cancer Survivorship Journey: Models of Care, Disparities, Barriers, and Future Directions. Am Soc Clin Oncol Educ book Am Soc Clin Oncol Meet 35:231-9, 2016

28. Khera N, Martin P, Edsall K, et al.: Patient-centered care coordination in hematopoietic cell transplantation. Blood Adv 1:1617-1627, 2017 [PubMed: 29296802] 


\section{CLINICAL PRACTICE POINTS}

Long-term survivors of hematologic malignancies and hematopoietic cell transplantation (HCT) frequently receive healthcare through their primary care physicians (PCPs) and are less likely to be under the care of cancer centers and hematologist-oncologists.

Barriers faced by PCPs in caring for this patient population have not been well described. Using a web-based survey of PCPs employed in two large integrated healthcare systems, we identify perceived barriers to delivery of care to hematologic malignancy/HCT survivors, resources available to care for cancer survivors, practices for care coordination with hematologist-oncologists, and preferred models of care delivery. Common barriers identified by PCPs included lack of resources to facilitate care (69\%), lack of awareness of screening/prevention guidelines (55\%) and psychosocial needs of survivors (65\%), inadequate time (65\%), and patient preference to follow up with their oncologists (66\%). They expressed confidence in caring for general medical issues (84\%) and general cancer screening (73\%), but preferred oncologists manage cancer related medical issues (42\%), screen for cancer recurrence (52\%) and secondary cancers (55\%). PCPs who had previously cared for larger number of hematologic malignancy/HCT survivors and those further out since graduation from medical school reported greater confidence in managing cancer related medical issues. Our study highlights several barriers faced by PCPs in providing care to hematologic malignancy/HCT survivors. However, several of these barriers are potentially addressable by education of PCPs focused on issues relevant to this patient population, development of evidence-based guidelines, implementation of clinical decision support tools, enhancing clinical experience, and better care coordination with hematologist-oncologists. 


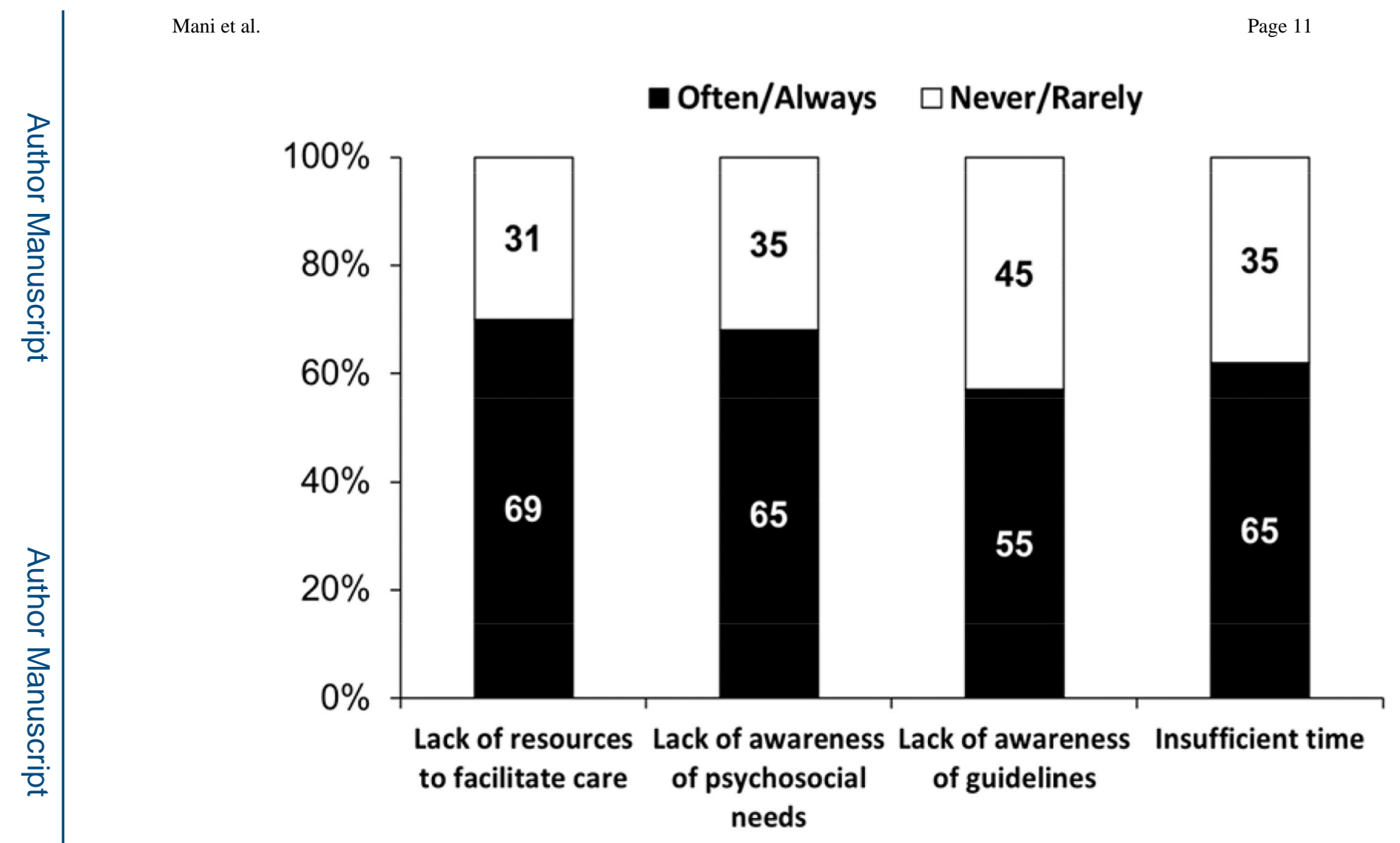

Fig. 1a.

Challenges in caring for cancer survivors in general 
- Often/Always $\square$ Never/Rarely

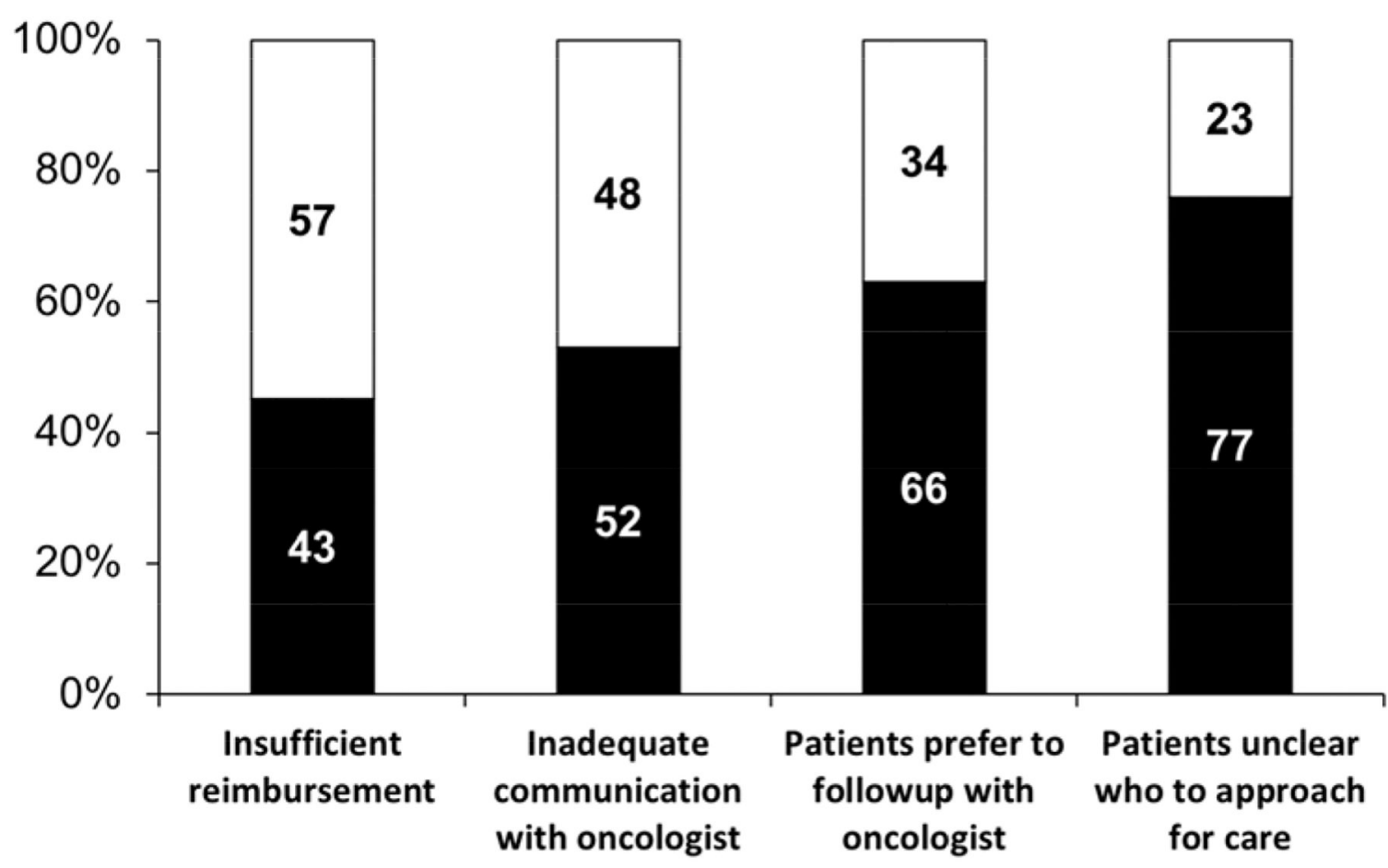

Fig. 1b.

Challenges in caring for cancer survivors in general 


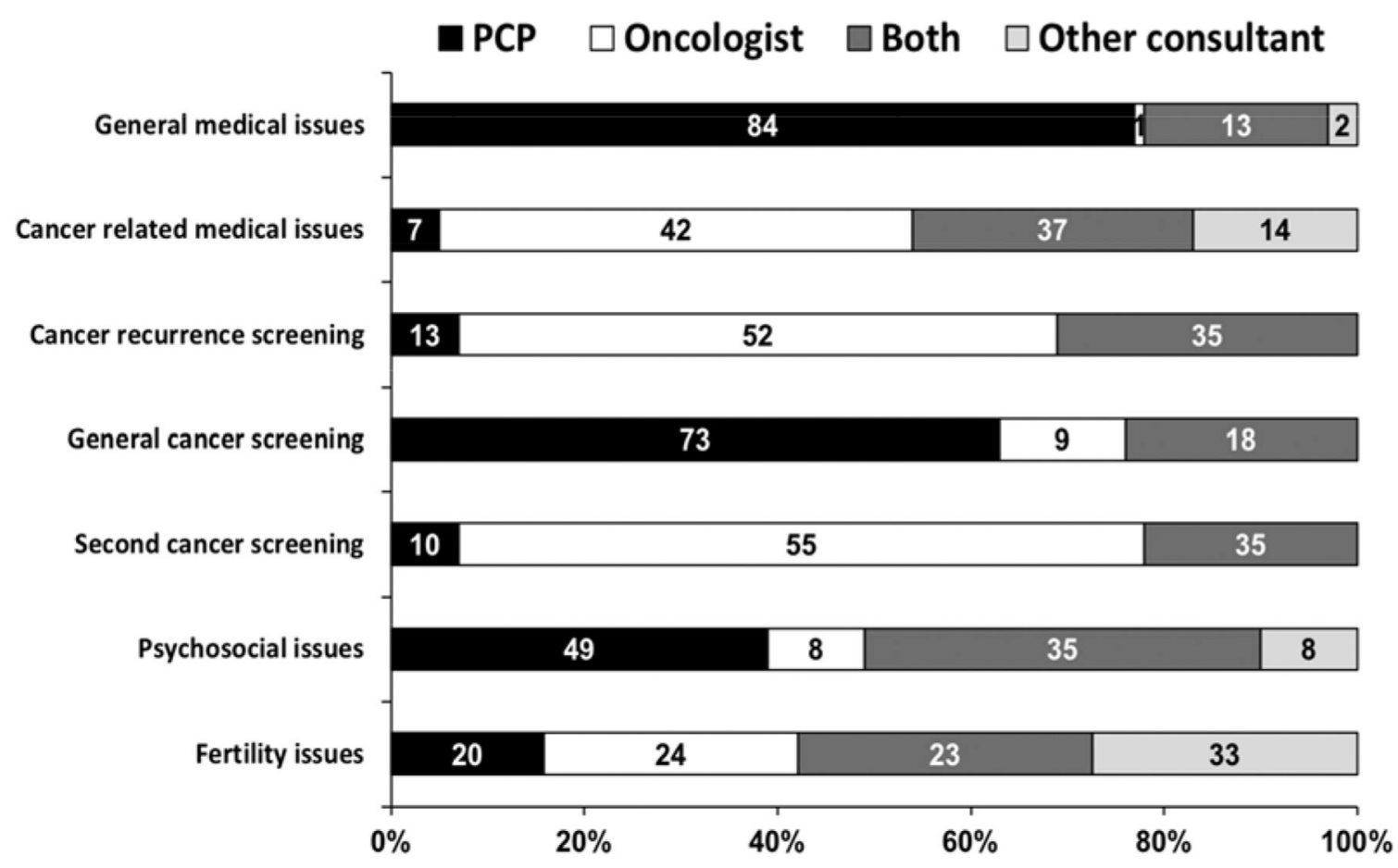

Fig. 1c.

Service provider preferences for providing care for hematologic malignancies and HCT survivors 


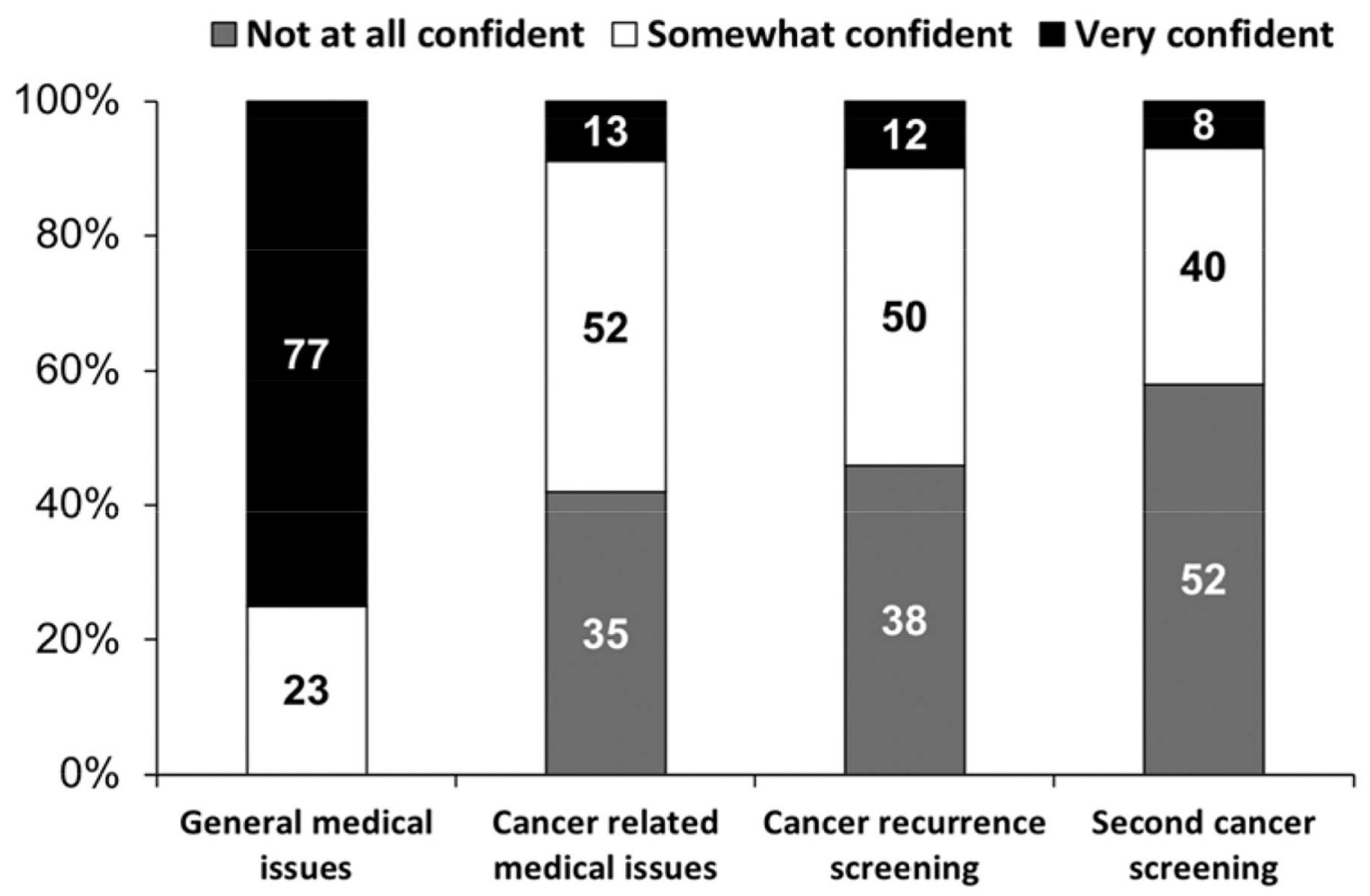

Fig. 2a.

Case scenario of patient with acute myeloid leukemia treated with chemotherapy only 


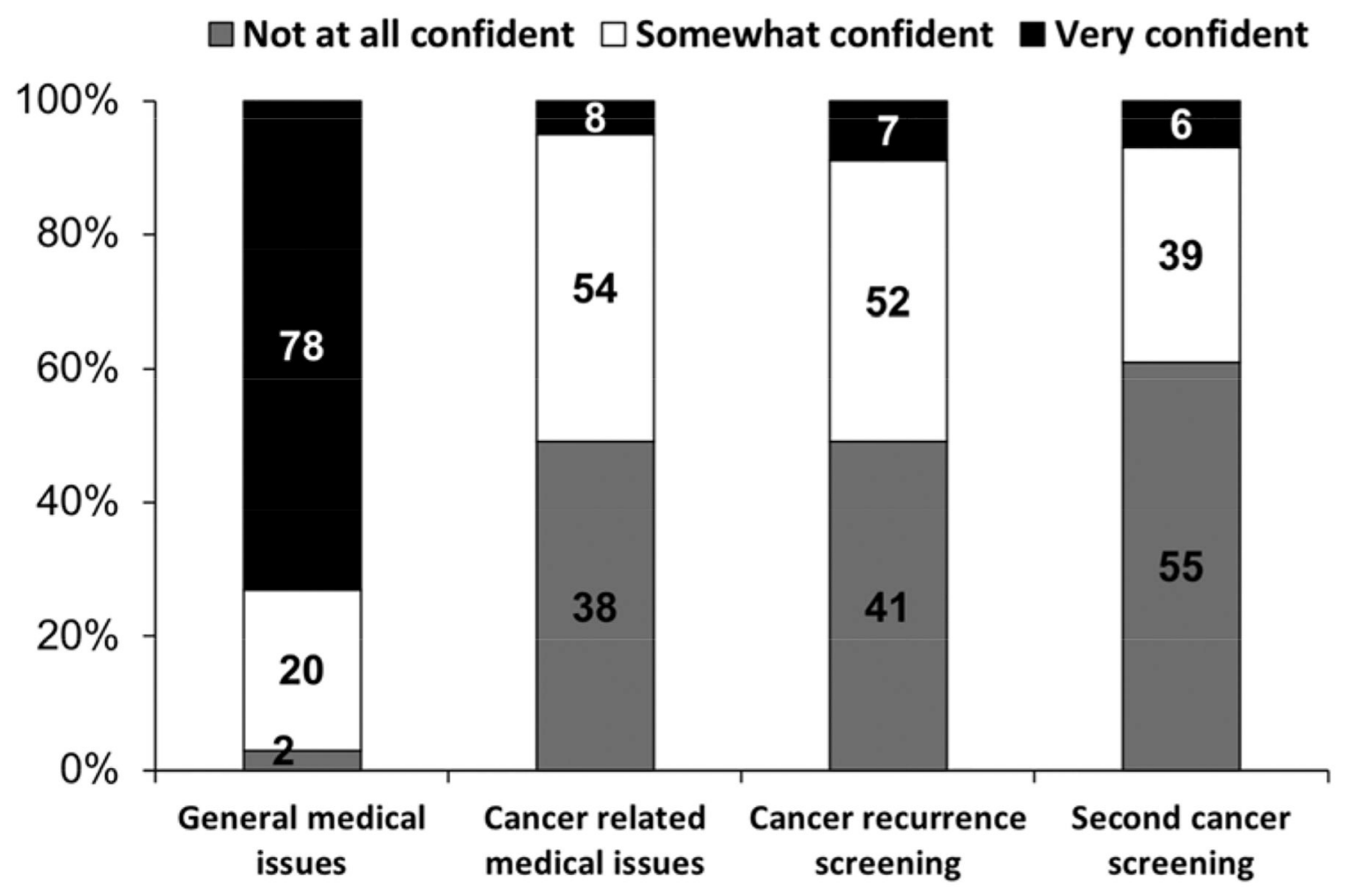

Fig. 2b.

Case scenario of patient with acute myeloid leukemia treated with an allogeneic HLAidentical sibling donor transplant. 
Table 1:

Characteristics of survey respondents

\begin{tabular}{|l|c|c|}
\hline \multicolumn{1}{|c|}{ Characteristic } & N & $\%$ \\
\hline Respondents & 86 & \\
\hline Institution & & \\
\hline Cleveland Clinic, Ohio & 59 & 69 \\
\hline Mayo Clinic, Arizona & 27 & 31 \\
\hline Primary site of practice & & \\
\hline Main campus & 34 & 40 \\
\hline Satellite clinic/hospital & 62 & 60 \\
\hline Primary specialty & & \\
\hline Internal Medicine & 60 & 70 \\
\hline Family Medicine & 21 & 24 \\
\hline Medicine-Pediatrics & 5 & 6 \\
\hline Years since graduation from medical school ${ }^{*}$ & & \\
\hline Median (range) & 17 & $1-47$ \\
\hline Currently teaching medical students or residents & & \\
\hline Yes & 68 & 79 \\
\hline No & 18 & 21 \\
\hline Number of patients seen in a typical week & & \\
\hline$<25$ & 9 & 11 \\
\hline $26-50$ & 32 & 21 \\
\hline $51-75$ & 19 & 21 \\
\hline $76-100$ & 9 & 11 \\
\hline$>100$ & 6 & 24 \\
\hline Number of hematologic malignancy/HCT survivors cared for in last 5 years & & \\
\hline 0 & 6 & 7 \\
\hline $1-5$ & 36 \\
\hline $6-10$ & & 21 \\
\hline $11-20$ & 21 \\
\hline$>20$ & 97 \\
\hline
\end{tabular}

HCT indicates hematopoietic cell transplantation

* $\mathrm{N}=85$ respondents 


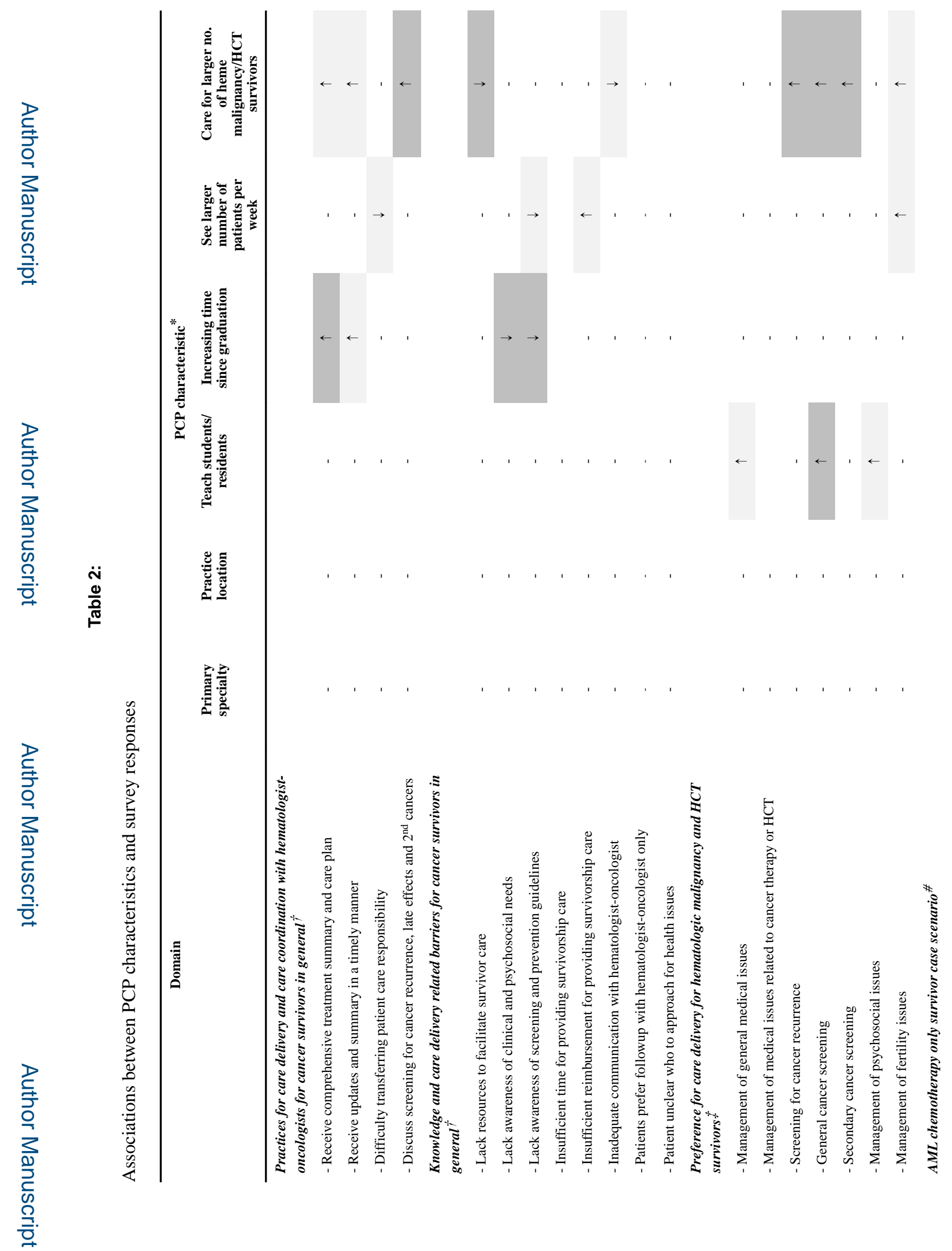

Clin Lymphoma Myeloma Leuk. Author manuscript; available in PMC 2021 February 01. 
Mani et al.

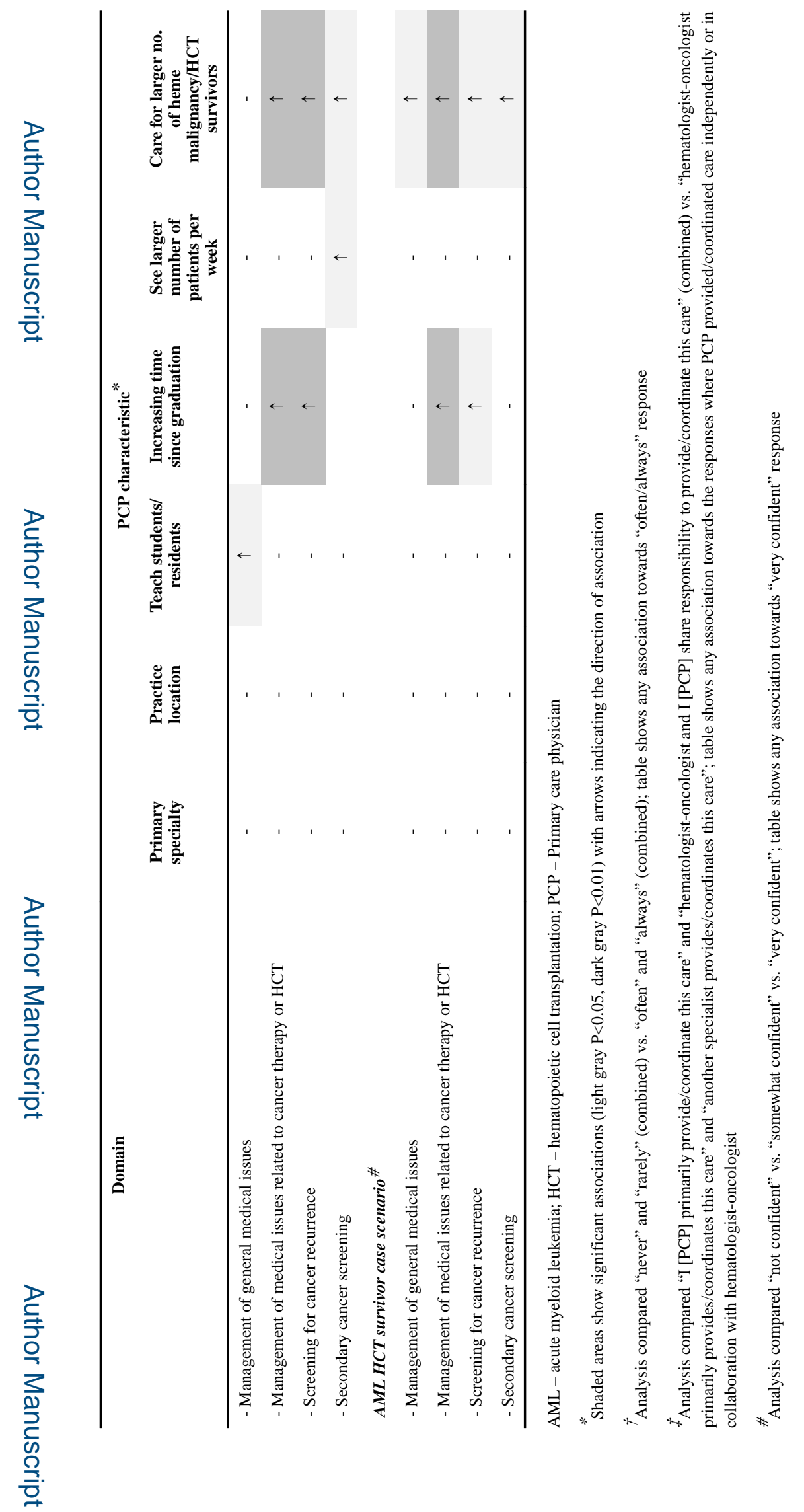

Clin Lymphoma Myeloma Leuk. Author manuscript; available in PMC 2021 February 01. 


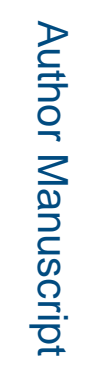

\begin{tabular}{|c|c|c|c|c|c|c|c|}
\hline 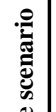 & 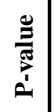 & & $\begin{array}{l}\text { :̊ } \\
\stackrel{0}{0}\end{array}$ & $\begin{array}{l}\mathscr{0} \\
\stackrel{0}{0} \\
\end{array}$ & $\begin{array}{l}\text { to } \\
0\end{array}$ & $\begin{array}{l}\stackrel{+}{\Delta} \\
\text { Oे }\end{array}$ & \\
\hline 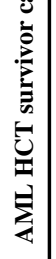 & $\begin{array}{l}0 \\
0 \\
0 \\
0 \\
0 \\
0 \\
0 \\
0 \\
0 \\
0 \\
0\end{array}$ & & 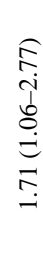 & 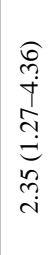 & 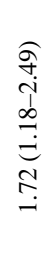 & 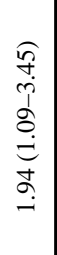 & \\
\hline 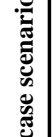 & 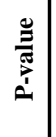 & $\stackrel{n}{0}$ & & $\frac{n}{a}$ & $\begin{array}{l}\bar{\delta} \\
\dot{\nabla}\end{array}$ & $\stackrel{0}{0}$ & 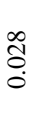 \\
\hline
\end{tabular}

政
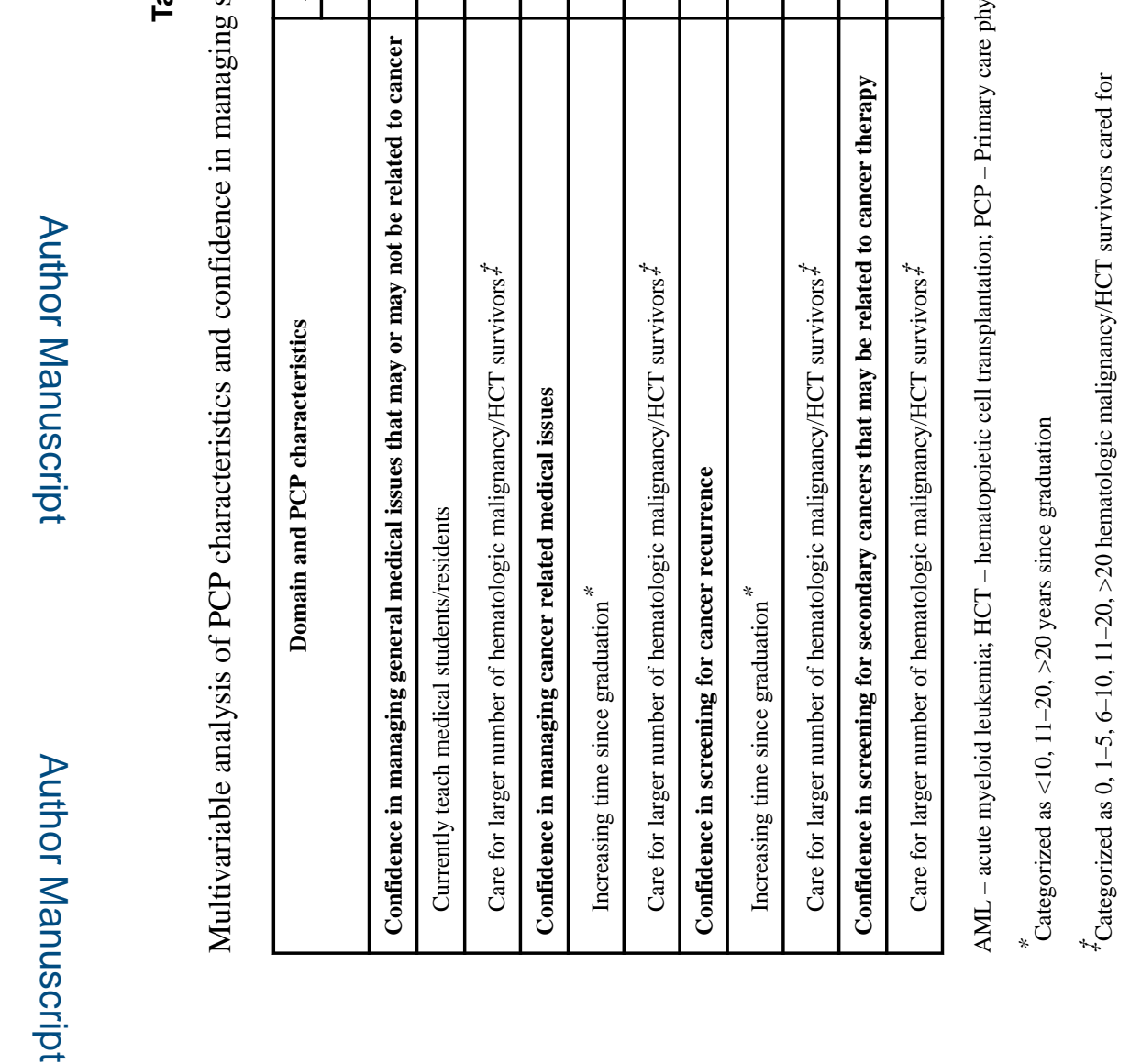

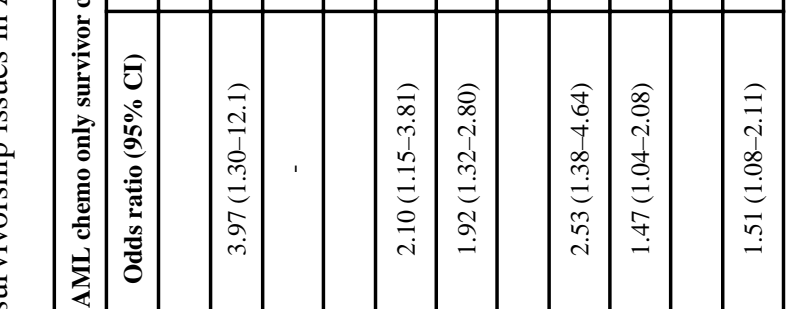

Clin Lymphoma Myeloma Leuk. Author manuscript; available in PMC 2021 February 01. 